\title{
Uso del polen de abeja en la alimentación de pollos de engorda
}

\author{
Use of bee pollen in broiler diet
}

\author{
Maria Cristina de Oliveiraa, Fernando Carlos Locha, Diones Montes da Silvab, Poliana \\ Carneiro Martinsc, Adriely Suzian Teixeirab, Daniel Cunha Claroa
}

\begin{abstract}
RESUMEN
El polen de abeja (PA) puede ser un complemento nutricional para los animales, ya que mejora la eficiencia del uso de nutrientes, lo que aumenta su absorción, acelera el crecimiento de los animales y mejora su rendimiento productivo. El efecto del PA en la dieta de pollos de engorda se evaluó sobre la digestibilidad, rendimiento, mucosa intestinal y calidad de la cama. Para evaluar la digestibilidad, se utilizaron 200 aves en un diseño de bloques al azar con cuatro tratamientos (0, 0.5, 1.0 y $1.5 \%$ de inclusión de PA) y cinco repeticiones. Cuatrocientas (400) aves se utilizaron para evaluar el desempeño, la morfología de la mucosa intestinal y calidad de la cama, en un diseño completamente al azar con los cuatro tratamientos y cinco repeticiones. El polen tuvo un efecto cuadrático en la digestibilidad aparente de la materia seca y extracto etéreo y un efecto lineal sobre la retención de calcio y en el valor de la energía metabolizable aparente. La inclusión de PA no influyó ( $P>0.05)$ en el desempeño, rendimiento de la canal y las vísceras o el duodeno y el yeyuno a los 42 días, el íleon a los 21 días y la calidad de la cama hasta 21 días de edad. El PA mejoró el rendimiento del páncreas, de vellosidades, la morfología en el duodeno y el yeyuno a los 21 días, y en el íleon a los 42 días y la volatilización de amoníaco. Como conclusión, incluyendo $\mathbf{1 . 5 \%}$ de polen de abeja mejora la digestibilidad de los nutrientes y la morfología intestinal, pero no el comportamiento productivo.
\end{abstract}

PALABRAS ClaVE: Aditivos, Nutrición, Pollos de engorda, Productos apícolas, Suplementación alimenticia.

\begin{abstract}
Bee pollen (BP) can be a nutritional complement for animals because it improves nutrient use efficiency, increasing their absorption and accelerating animal growth, improving their productive performance. The effect of BP in broiler diet was assessed on digestibility, performance, intestinal mucosa and litter quality. To assess the digestibility, 200 birds were used in a randomized block design with four treatments $(0,0.5,1$ and $1.5 \%$ BP inclusion) and five replications. Four hundred (400) birds were used to assess performance, intestinal mucosa morphology and litter quality, in a completely randomized design with the four treatments and five replications. BP had a quadratic effect on apparent digestibility of dry matter and ether extract and a linear effect on calcium retention and on apparent metabolizable energy value. Including BP did not influence ( $P>0.05)$ the performance, carcass yield, and viscera or the duodenum and the jejunum at $42 \mathrm{~d}$, the ileum at $21 \mathrm{~d}$ and litter quality up to $21 \mathrm{~d}$ of age. BP improved the pancreas yield, villus morphology in the duodenum and jejunum at $21 \mathrm{~d}$, and in the ileum at $42 \mathrm{~d}$ and ammonia volatilization. As conclusion, BP at $\mathbf{1 . 5} \%$ improves nutrient digestibility and intestinal morphology but not the productive performance.
\end{abstract}

KEY WORDS: Additives, Broiler nutrition, Bee products, Food supplements.

\section{INTRODUCCIÓN}

El polen de abeja (PA) es un aglomerado de polen de flores recogido por las abejas

\section{INTRODUCTION}

Bee pollen (BP) is an agglomerate of flower pollen collected by the bees mixed with nectar

\footnotetext{
Recibido el 5 de noviembre de 2014. Aceptado el 15 de enero de 2015.

a Faculdade de Medicina Veterinaria, Universidade de Rio Verde, Campus Universitário. Rio Verde, G0, Brasil. 75.901-910. mcorv@ig.com.br. Correspondencia al primer autor

b Departamento de Zootecnia, Instituto Federal Goiano, Rio Verde, GO, Brasil.

c Departamento de Produção Animal, Universidade Federal de Goiás, Goiânia, GO, Brasil.
} 
mezclados con néctar y la secreción de las glándulas hipofaríngeas(1) y es un suplemento biológicamente activo con influencia positiva en diversas funciones fisiológicas del organismo. Es rico en hidratos de carbono (2.6 a $22.4 \%$ ), proteína (15.01 a $36.73 \%$ ), aminoácidos, grasas (aproximadamente $9.2 \%$ ) donde el 60 al $91 \%$ son ácidos grasos insaturados(2), vitaminas $A$, $B, C, D$ y $E(3)$, minerales, carotenoides y flavonoides(4). Tiene acciones antibacteriales, antifúngicas, antinflamatorias y una acción inmunomoduladora, así como actividad antioxidante similar a los antioxidantes naturales como el $\alpha$-tocoferol, y superior a los sintéticos tales como el hidroxitolueno butilado(1), porque contiene sustancias polifenólicas, como los flavonoides, que secuestran los radicales libres que dañan la salud(5).

EI PA puede ser un complemento nutricional para los animales, ya que mejora la eficiencia en el uso de nutrientes, aumentando su absorción, acelera el crecimiento de los animales, y mejora su rendimiento productivo. Su uso en la dieta del pollo de engorda aumentó la longitud del duodeno, yeyuno e íleon durante los primeros 14 días de vida, lo que resulta en una área de absorción mayor y mejora la retención y digestibilidad de los nutrientes(4).

El uso del PA en la dieta de pollos de engorda resultó en aumento en el peso corporal de $35.1 \%$ de 1 a 42 días de edad(6), además de aumentar el peso del hígado y del páncreas(7). Pan et al(8) informaron que el uso de $0.2 \%$ de PA aumentó el peso corporal de las aves en la octava semana de vida sin alterar la conversión alimenticia.

El objetivo del presente estudio fue evaluar si con la inclusión de polen de abeja en la alimentación de pollos de engorda, se beneficiarían parámetros como la retención y la digestión de nutrientes, el rendimiento productivo, la canal, los cortes, el rendimiento de las vísceras, la morfología intestinal y la calidad de la cama de pollo. and secretions from the hypofaryngean glands(1) and is a biologically active supplement with positive influence on various physiological functions of the organism. It is rich in carbohydrates (2.6 to $22.4 \%$ ), protein (15.01 to $36.73 \%$ ), amino acids, fats (approximately $9.2 \%$ ) where 60 to $91 \%$ are unsaturated fatty acids(2), vitamins $A, B, C, D$ and $E(3)$, minerals, carotenoids and flavonoids(4). It has antibacterial, antifungal, anti-inflammatory and immunomodulating action and it has antioxidant activity similar to the natural antioxidants such as $\alpha$-tocopherol and superior to the synthetic ones such as butylated hydroxytoluene(1), because it has polyphenolic substances such as the flavonoids, that sequesters health-damaging free radicals(5).

BP can be a nutritional complement for animals because it improves nutrient use efficiency, increasing their absorption and accelerating animal growth, improving their productive performance. Use of BP in broiler chick diet increased the length of the duodenum, jejunum and ileum during the first $14 \mathrm{~d}$ of life, resulting in a greater absorption area and improving nutrient digestibility and retention(4).

Using BP in broiler chick diet resulted in increase in body weight from $35.1 \%$ from 1 to $42 \mathrm{~d}$ of age(6) in addition to increasing liver and pancreas weight(7). Pan et al(8) reported that using $0.2 \%$ BP increased body weight of the birds in the eighth week of life without altering feed conversion.

The objective of the present study was to assess whether including bee pollen in broiler diet would benefit nutrient digestion and retention, productive performance, carcass, cuts and viscera yield, intestinal morphology and chicken litter quality.

\section{MATERIALS AND METHODS}

Two hundred (200) male 13-d-old Cobb chicks were used in the digestibility trial, housed in metabolism cages equipped with linear drinkers 


\section{MATERIALES Y MÉTODOS}

Doscientos (200) pollitos Cobb machos de 13 días de edad se utilizaron en el ensayo de digestibilidad, alojados en jaulas metabólicas equipadas con bebederos y comederos lineales. El período experimental duró 10 días (seis para la adaptación a la dieta experimental en jaulas y cuatro para la recaudación total de la excreción). Se utilizó un diseño de bloques al azar, de acuerdo a la altura de las jaulas, con cuatro tratamientos y cinco repeticiones de 10 animales cada uno. Los tratamientos consistieron en niveles de PA en la dieta de 0, 0.5, 1 y $1.5 \%$.

La composición evaluada del PA utilizado fue, en porcentaje, 3.83 humedad, 22.97 de proteína cruda, calcio $0.39,0.99$ fósforo, la materia mineral 13.14 y 1.71 grasas; además de 3,953 $\mathrm{kcal} / \mathrm{kg}$ de energía cruda y $\mathrm{pH}$ de 4.68 .

Todas las aves recibieron la dieta y el agua libremente hasta los 12 días de edad, con una dieta isonutritiva, sin marcador o PA y se formularon de acuerdo con Rostagno et a/(9) a excepción de la proteína cruda (PC), energía metabolizable aparente (EMA), Ca y $\mathrm{P}$ niveles que corresponden a $97 \%$ de los niveles recomendados por los mismos autores. Hasta esta edad, las aves se criaron en un gallinero convencional. En el día 13 de vida, las aves, con un peso promedio de $389.69 \pm 7.04 \mathrm{~g}$, se alojaron en jaulas y comenzaron a recibir la dieta de los tratamientos respectivos. Se añadió óxido ferroso en polvo a las dietas ( $1 \%$ ) como indicador fecal en el inicio y el final de la colección de excretas. Se registró la ingesta de la dieta y la producción total de excretas, la cual se hizo a partir de los 20 a los 23 días de edad, en intervalos de $12 \mathrm{~h}$. Se identificó la excreción recogida, se almacenó en un congelador y luego se pre-secó en una cámara de ventilación forzada a $65{ }^{\circ} \mathrm{C}$ durante $48 \mathrm{~h}$ y se molió.

Todos los análisis de la excreción, dietas y PA se llevaron a cabo según lo informado por Silva y Queiroz $(10)$. Una vez que se obtuvieron los and feeders. The experimental period lasted for $10 \mathrm{~d}$ (six for adaptation to the experimental diet and cages and four for total excretion collection). A randomized block design was used, placed according to the height of the cage stories, with four treatments and five replications of 10 birds each. The treatments consisted of ground BP levels in the broiler chick diet $(0$, $0.5,1.0$ and $1.5 \%)$.

The determined composition of the BP used was, in percentage, 3.83 moisture, 22.97 crude protein, 0.39 calcium, 0.99 phosphorus, 3.14 mineral matter and 1.71 fats, in addition to $3,953 \mathrm{kcal} / \mathrm{kg}$ crude energy and $\mathrm{pH} 4.68$.

All the birds received diet and water freely up to $12-d$ of age. The isonutritive diets did not contain marker or BP and were formulated according to Rostagno et a/(9) except for the crude protein (CP), apparent metabolizable energy (AME), $\mathrm{Ca}$ and $\mathrm{P}$ levels that corresponded to $97 \%$ of the levels recommended by the same authors. Up to this age, the birds were raised in a conventional chicken shed. On the 13th day of life, the birds, $389.69 \pm 7.04 \mathrm{~g}$ mean weight, housed in cages and began receiving the diet of the respective treatments. Ferric oxide in powder was added to the diets $(1 \%)$ as fecal indicator at the start and end of the excretion collection. The diet intake and total excretion production were recorded. Total excretion collection was made from the $20^{\text {th }}$ to the $23^{\text {rd }}$ day of age, at $12 \mathrm{~h}$ intervals. The excretion collected was identified, stored in a freezer and then pre-dried in a forced ventilation chamber at $65{ }^{\circ} \mathrm{C}$ for $48 \mathrm{~h}$ and ground.

All the analyses of the excretion, diets and BP were carried out as reported by Silva and Queiroz(10). Once the results of laboratory analyses had been obtained, the coefficients of apparent digestibility (CD), dry matter (DM), crude protein (CP) and ether extract (EE), calcium (Ca) and phosphorus retention (P) and the apparent metabolizable energy (AME) and apparent metabolizable energy corrected (AMEn) by the nitrogen balance were calculated. 
resultados de los análisis de laboratorio, se calcularon los coeficientes de digestibilidad aparente $(C D)$ de la materia seca (MS), proteína cruda $(\mathrm{PC})$, extracto de etéreo (EE), calcio $(\mathrm{Ca})$, retención de fósforo aparente $(P)$, energía metabolizable aparente (EMA) y la energía metabolizable aparente corregida (EMAn) por el balance de nitrógeno.

En el ensayo de rendimiento, se utilizaron 400 pollitos, machos (M) y hembras $(\mathrm{H})$ de un día de edad, con peso inicial promedio de $52.44 \pm$ $1.76 \mathrm{~g}$. Las aves se alojaron en un cobertizo de mampostería dividido en casillas experimentales de $1.85 \mathrm{~m}^{2}$ con una densidad de 10.8 aves $/ \mathrm{m}^{2}$.

Se utilizó un diseño completamente al azar con cuatro tratamientos y cinco repeticiones de 12 aves ( $\mathrm{M}$ y $\mathrm{H}$ ) por unidad experimental, y los tratamientos consistieron en aumentar los niveles de PA en la dieta de los polluelos de engorda, que fueron $0,0.5,1$ y $1.5 \%$. Las aves recibieron una dieta de inicio desde el primer día hasta los 21 días, y otra en crecimiento de 22 a 42 días de edad (Cuadro $1)$.

Las dietas experimentales fueron isonutritivas y formuladas para satisfacer las necesidades del pollo de engorda(9), a excepción de los niveles de $C P, A M E$, Ca y $P$ que correspondían al $97 \%$ de los niveles recomendados por los mismos autores. El agua y la dieta se suministraron a libertad durante todo el período experimental.

Las aves se pesaron a los 21 y 42 días de edad para determinar el aumento de peso. Las dietas también se pesaron para calcular la ingesta y la conversión alimenticia. También se determinó la tasa de supervivencia.

Al final de cada fase, dos aves por compartimento, de ambos sexos, previo ayuno durante $12 \mathrm{~h}$, se sacrificaron por desplazamiento cervical. De cada ave se obtuvieron dos muestras de $2 \mathrm{~cm}$ de la región del duodeno, en la parte media entre la entrada del conducto biliar y el divertículo de Meckel (yeyuno) y $10 \mathrm{~cm}$ de la
In the performance trial, 400 one-day-old chicks were used, male (M) and female (F) with mean initial weight $52.44 \pm 1.76 \mathrm{~g}$. The birds were housed in a masonry shed divided into $1.85 \mathrm{~m}^{2}$ experimental boxes at a density of 10.8 birds/square meter.

A complete randomized design was used with four treatments and five replications of 12 birds ( $M$ and $F$ ) per experimental unit and treatments consisted of increasing ground BP levels in the broiler chick diet. The inclusion levels assessed were 0, 0.5, 1 and $1.5 \%$. The birds received initial diet starting on the first day until d 21 and growing diet from 22 to $42 \mathrm{~d}$ of age (Table 1 ).

The experimental feeds were isonutritive and formulated to meet broiler chick requirements(9), except for levels of $C P, A M E, C a$ and $P$ that corresponded $97 \%$ of the levels recommended by the same authors. Water and diet were supplied freely throughout the experimental period.

The birds were weighed at 21 and $42 \mathrm{~d}$ of age to determine weight gain. The diets were also weighed to calculate diet intake and feed conversion. The survival rate was also determined.

At the end of each phase, two birds per box, both sexes, were food fasted for $12 \mathrm{~h}$ and sacrificed by cervical displacement. Two 2-cm samples were collected from each bird in the region of the duodenum strap, in the half between the bile duct entry and the Meckel diverticulum (jejunum) and $10 \mathrm{~cm}$ from the ileum-cecum junction (ileum). The segments obtained were opened and fixed in $10 \%$ formaldehyde solution for $24 \mathrm{~h}$. The fragments were then processed for blocking in paraffin. Seried $5 \mu \mathrm{m}$ cuts were made in each fragment, stained with hematoxylin and eosine. Thirty (30) readings were made per fragment for villus height, perimeter and width, and crypt depth. The absorption surface (AS) was 
Cuadro 1. Composición calculada de dietas conteniendo polen de abeja (BP)

Table 1. Calculated composition of diets containing bee pollen (BP)

\begin{tabular}{|c|c|c|c|c|c|c|c|c|}
\hline \multirow[b]{2}{*}{ Ingredients (kg) } & \multicolumn{4}{|c|}{$\begin{array}{c}\text { Initial diet } \\
\text { BP level (\%) }\end{array}$} & \multicolumn{4}{|c|}{$\begin{array}{l}\text { Growth diet } \\
\text { BP level (\%) }\end{array}$} \\
\hline & 0.0 & 0.5 & 1.0 & 1.5 & 0.0 & 0.5 & 1.0 & 1.5 \\
\hline Ground corn & 55.00 & 55.00 & 55.00 & 55.00 & 59.05 & 59.05 & 59.05 & 59.05 \\
\hline Soybean meal & 36.24 & 36.24 & 36.24 & 36.24 & 32.00 & 32.00 & 32.00 & 32.00 \\
\hline Soybean oil & 3.10 & 3.10 & 3.10 & 3.10 & 3.89 & 3.89 & 3.89 & 3.89 \\
\hline Dicalcium phosphate & 1.92 & 1.92 & 1.92 & 1.92 & 1.68 & 1.68 & 1.68 & 1.68 \\
\hline Limestone & 0.94 & 0.94 & 0.94 & 0.94 & 0.80 & 0.80 & 0.80 & 0.80 \\
\hline L-Lysine 99\% & 0.20 & 0.20 & 0.20 & 0.20 & 0.05 & 0.05 & 0.05 & 0.05 \\
\hline DL-Methionine $99 \%$ & 0.20 & 0.20 & 0.20 & 0.20 & 0.13 & 0.13 & 0.13 & 0.13 \\
\hline Common salt & 0.50 & 0.50 & 0.50 & 0.50 & 0.50 & 0.50 & 0.50 & 0.50 \\
\hline Mineral/vitamin supplement & $0.40^{1}$ & $0.40^{1}$ & $0.40^{1}$ & $0.40^{1}$ & $0.40^{2}$ & $0.40^{2}$ & $0.40^{2}$ & $0.40^{2}$ \\
\hline Bee pollen & 0.00 & 0.50 & 1.00 & 1.50 & 0.00 & 0.50 & 1.00 & 1.50 \\
\hline Inert (caolim) & 1.50 & 1.00 & 0.50 & 0.00 & 1.50 & 1.00 & 0.50 & 0.00 \\
\hline Total & 100 & 100 & 100 & 100 & 100 & 100 & 100 & 100 \\
\hline \multicolumn{9}{|l|}{ Calculated composition 3} \\
\hline Crude protein, \% & 20.7 & 20.7 & 20.7 & 20.7 & 19.1 & 19.1 & 19.1 & 19.1 \\
\hline Metabolizable energy, kcal/kg & 2950 & 2950 & 2950 & 2950 & 3050 & 3050 & 3050 & 3050 \\
\hline Calcium, \% & 0.93 & 0.93 & 0.93 & 0.93 & 0.81 & 0.81 & 0.81 & 0.81 \\
\hline Available phosphorus, \% & 0.47 & 0.47 & 0.47 & 0.47 & 0.42 & 0.42 & 0.42 & 0.42 \\
\hline Sodium, \% & 0.22 & 0.22 & 0.22 & 0.22 & 0.22 & 0.22 & 0.22 & 0.22 \\
\hline Total lysine, \% & 1.34 & 1.34 & 1.34 & 1.34 & 1.08 & 1.08 & 1.08 & 1.08 \\
\hline Total methionine, $\%$ & 0.51 & 0.51 & 0.51 & 0.51 & 0.42 & 0.42 & 0.42 & 0.42 \\
\hline Total methionine+cystine, $\%$ & 0.84 & 0.84 & 0.84 & 0.84 & 0.73 & 0.73 & 0.73 & 0.73 \\
\hline
\end{tabular}

1 Each kg contains: Vitamin A 1500000 UI, Vitamin $D_{3} 375000$ Ul, Vitamin E 5000 UI, Vitamin $K_{3} 375$ mg, Vitamin $B_{1} 500$ mg, Vitamin $B_{2} 1625 \mathrm{mg}$, Vitamin $B_{6} 750 \mathrm{mg}$, Vitamin $B_{12} 3750 \mathrm{mcg}$, nicotinic acid $10000 \mathrm{mg}$, biotin $20 \mathrm{mg}$, folic acid $250 \mathrm{mg}$, calcium pantothenate 2500 mg, choline 65000 mg, methionine 450 g, Cu 2000 mg, Fe 8000 mg, I 250 mg, Mn 20000 mg, Se 75 mg, Zn $16500 \mathrm{mg}$, BHT $60 \mathrm{mg}$, avilamycin $2500 \mathrm{mg}$, nicarbazin $25000 \mathrm{mg}$.

2 Each kg contains: Vitamin A 1125000 UI, Vitamin $D_{3} 300000$ UI, Vitamin E 3750 UI, Vitamin $K_{3} 250$ mg, Vitamin B 450 mg, Vitamin $B_{2} 1125 \mathrm{mg}$, Vitamin $B_{6} 500 \mathrm{mg}$, Vitamin $B_{12} 2500 \mathrm{mcg}$, niacin $3750 \mathrm{mg}$, folic acid 125 mg, calcium pantothenate $2500 \mathrm{mg}$, choline $65000 \mathrm{mg}$, methionine $440 \mathrm{~g}$, Cu $2000 \mathrm{mg}$, Fe 8000 mg, I 250 mg, Mn 20000 mg, Se 75 mg, Zn 16500 $\mathrm{mg}$, BHT $60 \mathrm{mg}$, avilamycin $1875 \mathrm{mg}$, salinomycin $16500 \mathrm{mg}$.

3 According to Rostagno et al.(9).

unión íleo-ciego (íleon). Los segmentos obtenidos se abrieron y se fijaron en solución de formaldehído al $10 \%$ durante $24 \mathrm{~h}$. Los fragmentos se procesaron en bloques de parafina. Se hicieron cortes seriados de 5 micras en cada fragmento, se tiñeron con hematoxilina y eosina. Se realizaron 30 lecturas por fragmento de la altura de las vellosidades, perímetro y anchura, y profundidad de las criptas. La calculated according to the formula: $\mathrm{AS}\left(\mathrm{mm}^{2}\right)=$ villus height $(\mathrm{mm}) \times$ width at $50 \%$ villus height $(\mathrm{mm})$.

At $42 \mathrm{~d}$ two birds from each box were fasted and after $8 \mathrm{~h}$ were weighed, slaughtered and eviscerated and the carcass, cuts and the viscera yields were assessed. The slaughter weight was used as reference to calculate carcass and cut 
superficie de absorción (AS) se calculó según la fórmula: AS $\left(\mathrm{mm}^{2}\right)$ = altura de las vellosidades $(\mathrm{mm}) \times$ anchura al $50 \%$ de la altura de las vellosidades $(\mathrm{mm})$.

A los 42 días, dos aves de cada casilla se mantuvieron en ayunas y después de $8 \mathrm{~h}$ fueron pesadas, sacrificadas y evisceradas; se evaluaron la canal, cortes y los rendimientos de vísceras. El peso a la matanza se utilizó como referencia para calcular el rendimiento de la canal y el rendimiento de cortes, y el peso de la canal se utilizó como referencia para el rendimiento de vísceras.

La cáscara de arroz se utilizó como material de cama en todos los tratamientos, en cantidad de $10 \mathrm{~kg} /$ casilla. Para analizar la materia seca, nitrógeno total, fósforo(10), amoniaco volatilizado(11) y el pH del lecho, a los 21 y 42 días de cría se recogieron muestras en seis puntos dentro de cada casilla, evitando las zonas cerca y por debajo de comederos y bebederos, los resultados se sometieron a análisis de yield and the carcass weight was reference for the viscera yields.

Rice husk was used as bed material in all the treatments, at the quantity of $10 \mathrm{~kg} / \mathrm{box}$. To analyze dry matter, total nitrogen and phosphorus(10), volatized ammonia(11) and litter $\mathrm{pH}$, samples were collected at six points inside each box, avoiding areas close and below feeders and drinkers, at 21 and $42 \mathrm{~d}$ of rearing.

The results were submitted to analyses of variance and polynomial regression, when $F$ test was significant, the system of statistical and genetic analyses at 5\% probability level was performed.

\section{RESULTS}

Including BP in the diet had a quadratic effect on the digestibility coefficient of dry matter (DCDM) $(P<0.007)$ and digestibility coefficient of ether extract (DCEE) $(P<0.007)$ and a linear effect $(P<0.0001)$ on the AME and AMEn values and $\mathrm{Ca}$ retention $(P<0.048)$ (Table 2$)$.

Cuadro 2. Coeficientes de digestibilidad aparente de nutrientes, retención de Ca y P y energía metabolizable aparente (AME) y corregida (AMEn) de la dieta que contiene polen de abeja (BP)

Table 2. Coefficients of apparent digestibility of nutrients, $\mathrm{Ca}$ and $\mathrm{P}$ retention and apparent (AME) and corrected apparent (AMEn) metabolizable energy values of diet containing bee pollen (BP)

\begin{tabular}{lccccc}
\hline & \multicolumn{4}{c}{ BP level (\%) } & \\
\cline { 2 - 5 } & 0.0 & 0.5 & 1.0 & 1.5 & CV1 $(\%)$ \\
\hline Dry matter, \% & 73.53 & 73.64 & 73.75 & 76.54 & 1.55 \\
Crude protein, \% & 60.64 & 67.41 & 66.86 & 70.26 & 4.71 \\
Ether extract, \%3 & 87.64 & 87.86 & 88.16 & 89.67 & 1.43 \\
Calcium retention, \%4 & 72.13 & 77.91 & 75.63 & 80.83 & 4.44 \\
Phosphorus retention, \% & 63.37 & 64.26 & 62.65 & 68.21 & 4.94 \\
AME, kcal/kg5 & 3139 & 3154 & 3187 & 3383 & 1.95 \\
AMEn, kcal/kg6 & 3079 & 3092 & 3128 & 3321 & 1.85 \\
\hline
\end{tabular}

1 Coefficient of variation.

2 Quadratic effect $\left(\hat{Y}=73.67-2.19 x+2.68 x^{2} ; R^{2}=0.58\right)$.

3 Quadratic effect $\left(\hat{Y}=87.70-0.65 x+1.29 x^{2} ; R^{2}=0.60\right)$.

4 Linear effect $\left(\hat{Y}=73.06+4.76 x r^{2}=0.25\right)$.

5 Linear effect $\left(\hat{Y}=3101+153 x ; r^{2}=0.76\right)$.

6 Linear effect $\left(\hat{Y}=3041+152 x ; r^{2}=0.76\right)$. 
Cuadro 3. Morfología del duodeno de pollos de engorda alimentados con dietas con diversos niveles de polen de abeja (BP) a los 21 y 42 días de edad

Table 3. Duodenum morphology of broiler chicks fed diets containing levels of bee pollen (BP) at 21 and $42 \mathrm{~d}$ of age

\begin{tabular}{|c|c|c|c|c|c|}
\hline & \multicolumn{4}{|c|}{ BP level (\%) } & \multirow[b]{2}{*}{$\mathrm{CV}^{1}(\%)$} \\
\hline & 0.0 & 0.5 & 1.0 & 1.5 & \\
\hline \multicolumn{6}{|c|}{21 days } \\
\hline Villus height, $\mu \mathrm{m}^{2}$ & 1273 & 1466 & 1521 & 1441 & 5.42 \\
\hline Villus perimeter, $\mu \mathrm{m}^{3}$ & 2593 & 2999 & 3164 & 2952 & 5.21 \\
\hline Villus width, $\mu \mathrm{m}$ & 87 & 75 & 83 & 98 & 5.52 \\
\hline Crypt depth, $\mu \mathrm{m}$ & 98 & 123 & 174 & 152 & 4.12 \\
\hline Absorption surface, $\mathrm{mm}^{24}$ & 0.111 & 0.110 & 0.127 & 0.142 & 7.01 \\
\hline \multicolumn{6}{|c|}{42 days } \\
\hline Villus height, $\mu \mathrm{m}$ & 1766 & 1753 & 1688 & 1727 & 2,76 \\
\hline Villus perimeter, $\mu \mathrm{m}$ & 3393 & 3087 & 3381 & 3436 & 2,64 \\
\hline Villus width, $\mu \mathrm{m}$ & 135 & 170 & 157 & 125 & 4,78 \\
\hline Crypt depth, $\mu \mathrm{m}$ & 97 & 103 & 117 & 112 & 6,73 \\
\hline Absorption surface, $\mathrm{mm}^{2}$ & 0,231 & 0,314 & 0,494 & 0,217 & 9,72 \\
\hline
\end{tabular}

$1 \mathrm{CV}=$ Coefficient of variation, obtained with transformed mean $-\log (X+1)$.

2 Quadratic effect ( $\left.\hat{Y}=1273.35+520.76 x-272.6 x^{2} ; R^{2}=0.66\right)$.

3 Quadratic effect $\left(\hat{Y}=2585.98+1177.55 x-619.21 x^{2} ; R^{2}=0.71\right)$.

4 Linear effect $\left(\hat{Y}=0.106+0.022 x ; r^{2}=0.30\right)$.

Cuadro 4. Morfología del yeyuno de pollos de engorda alimentados con dietas con diversos niveles de polen de abeja (BP) a los 21 y 42 días de edad

Table 4. Jejunum morphology of broiler chicks fed diets containing levels of bee pollen (BP) at 21 and $42 \mathrm{~d}$ of age

\begin{tabular}{lccccc}
\hline & \multicolumn{4}{c}{ BP level (\%) } & \\
\cline { 2 - 5 } & 0.0 & 0.5 & 1.0 & 1.5 & CV $^{1}(\%)$ \\
\hline & & 21 days & & & \\
Villus height, $\mu \mathrm{m}^{2}$ & 841 & 930 & 1059 & 976 & 1.49 \\
Villus perimeter, $\mu \mathrm{m}^{3}$ & 1762 & 1939 & 2147 & 2055 & 1.47 \\
Villus width, $\mu \mathrm{m}^{4}$ & 91 & 70 & 71 & 86 & 2.92 \\
Crypt depth, $\mu \mathrm{m}$ & 100 & 103 & 95 & 107 & 3.82 \\
Absorption surface, $\mathrm{mm}^{25} 5$ & 0.074 & 0.065 & 0.075 & 0.084 & 8.07 \\
& & 42 days & & & \\
Villus height, $\mu \mathrm{m}$ & 1265 & 1218 & 1577 & 1161 & 2.95 \\
Villus perimeter, $\mu \mathrm{m}$ & 2573 & 2452 & 3137 & 2330 & 2.75 \\
Villus width, $\mu \mathrm{m}$ & 148 & 131 & 118 & 145 & 4.33 \\
Crypt depth, $\mu \mathrm{m}$ & 141 & 158 & 142 & 141 & 3.93 \\
Absorption surface, $\mathrm{mm}^{2}$ & 0.184 & 0.159 & 0.187 & 0.101 & 7.04 \\
\hline
\end{tabular}

$1 \mathrm{CV}=$ coefficient of variation, obtained with transformed mean $-\log (X+1)$.

2 Quadratic effect $\left(\hat{Y}=827.93+367.37 x-173.66 x^{2} ; R^{2}=0.47\right)$

3 Quadratic effect $\left(\hat{Y}=1745.71+621.00 x-269.33 x^{2} ; R^{2}=0.40\right)$

4 Quadratic effect $\left(\hat{Y}=90.18-55.73 x+35.58 x^{2} ; R^{2}=0.48\right)$

5 Quadratic effect $\left(\hat{Y}=0.073-0.019 x+0.018 x^{2} ; R^{2}=0.54\right)$ 
María Cristina de Oliveira, et al. / Rev Mex Cienc Pecu 2015;6(3):263-276

Cuadro 5. Morfología ileal de pollos de engorda alimentados con dietas con diversos niveles de polen de abeja (BP) a los 21 y 42 días de edad

Table 5. Ileal morphology of broiler chicks fed diets containing levels of bee pollen (BP) at 21 and $42 \mathrm{~d}$ of age

\begin{tabular}{|c|c|c|c|c|c|}
\hline & \multicolumn{4}{|c|}{ BP level (\%) } & \multirow[b]{2}{*}{$\mathrm{CV}^{1}(\%)$} \\
\hline & 0.0 & 0.5 & 1.0 & $\overline{1.5}$ & \\
\hline \multicolumn{6}{|c|}{21 days } \\
\hline Villus height, $\mu \mathrm{m}$ & 703 & 748 & 629 & 613 & 3.21 \\
\hline Villus perimeter, $\mu \mathrm{m}$ & 1471 & 1562 & 1319 & 1266 & 2.55 \\
\hline Villus width, $\mu \mathrm{m}$ & 109 & 87 & 140 & 121 & 4.96 \\
\hline Crypt depth, $\mu \mathrm{m}$ & 105 & 108 & 100 & 101 & 5.55 \\
\hline Absorption surface, $\mathrm{mm}^{2}$ & 0.074 & 0.064 & 0.093 & 0.073 & 6.32 \\
\hline \multicolumn{6}{|c|}{42 days } \\
\hline Villus height, $\mu \mathrm{m}^{2}$ & 823 & 1049 & 1287 & 997 & 2.82 \\
\hline Villus perimeter, $\mu \mathrm{m}^{3}$ & 1770 & 2216 & 2663 & 2017 & 2.38 \\
\hline Villus width, $\mu \mathrm{m}$ & 218 & 184 & 149 & 203 & 2.88 \\
\hline Crypt depth, $\mu \mathrm{m}$ & 125 & 135 & 158 & 120 & 4.57 \\
\hline Absorption surface, $\mathrm{mm}^{2}$ & 0.177 & 0.188 & 0.191 & 0.201 & 9.86 \\
\hline
\end{tabular}

varianza y regresión polinómica; cuando la prueba de $\mathrm{F}$ fue significativa, se utilizó el sistema de análisis estadístico y genético a nivel de probabilidad del $5 \%$.

\section{RESULTADOS}

Incluyendo PA en la dieta tuvo un efecto cuadrático en el coeficiente de digestibilidad de la materia seca; CDMS $(P<0.007)$ y en el coeficiente de digestibilidad del extracto etéreo, CDEE $(P<0.007)$, y un efecto lineal $(P<0.001)$ en los valores de EMA, EMAn y retención de Ca $(P<0.048)$ (Cuadro 2).

Incluyendo PA en las dietas no influyó ( $P>0.05)$ en la morfometría del duodeno y la mucosa del yeyuno a los 42 días y en la mucosa del íleon a los 21 días. Sin embargo, la altura de las vellosidades y el perímetro y superficie de absorción del duodeno a los 21 días, se afectaron en forma cuadrática $(P<0.02)$ y lineal $(P<0.001)$, respectivamente. La altura de las
Including BP in the broiler chick diets did not influence $(P>0.05)$ the morphometry of the duodenum and jejunum mucosa at $42 \mathrm{~d}$ and the ileum mucosa at $21 \mathrm{~d}$. However, villus height and perimeter and duodenum absorption surface, at $21 \mathrm{~d}$, were affected quadratically $(P<0.02)$ and linearly $(P<0.001)$, respectively. The villi height, perimeter and width and the jejunum absorption surface at $21 \mathrm{~d}$ were affected quadratically $(P<0.03)$ and the ileum villus width and perimeter at $42 \mathrm{~d}$ were affected quadratically $(P<0.044)$ (Tables $3,4,5)$.

However, in spite of beneficially affect the digestibility of some nutrients and increasing the AME values, BP inclusion at 1 to $42 \mathrm{~d}$ of age did not influence $(P>0.05)$ the productive performance of the birds (Table 6 ); the carcass yields, noble cuts and edible viscera (Table 7). There was a quadratic effect on the pancreas $(P<0.03)$ where the highest values were obtained with inclusion of $1.5 \%$ BP. 
vellosidades, el perímetro y la anchura y la superficie de absorción del yeyuno a los 21 días se afectaron cuadráticamente $(P<0.03)$ y la anchura de las vellosidades del íleon y el perímetro a los 42 días se afectaron también cuadráticamente $(P<0.044)$ (Cuadros $3,4,5)$.

Sin embargo, a pesar de que beneficia la digestibilidad de algunos nutrientes y el aumento de los valores de AME, la inclusión de PA en la dieta no influyó $(P>0.05)$ el comportamiento
Including BP in the broiler chick diet had a quadratic effect $(P<0.014)$ on the quantity of ammonia volatized from the chicken litter at $42 \mathrm{~d}$ of rearing, but the other parameters were not affected $(P>0.05)$ at 21 and $42 \mathrm{~d}$ (Table 8).

\section{DISCUSSION}

The highest values of apparent digestibility of dry matter (ADDM), apparent digestibility of ether extract (ADEE), Ca retention, AME and

Cuadro 6. Rendimiento de pollos de engorda alimentados con dietas con diversos niveles de polen de abeja (BP)

Table 6. Performance of broiler chicks fed diets containing levels of bee pollen (BP)

\begin{tabular}{lccccc}
\hline & \multicolumn{5}{c}{ BP level (\%) } \\
\cline { 2 - 5 } & 0.0 & 0.5 & 1.0 & 1.5 & CV (\%) \\
\hline Weight gain, g & 554 & 688 & 609 & 647 & 6.71 \\
Feed intake, g & 755 & 776 & 779 & 801 & 6.24 \\
Feed:gain ratio & 1.36 & 1.13 & 1.28 & 1.24 & 4.83 \\
Livability rate, \% & 97.78 & 98.33 & 97.78 & 98.00 & 4.73 \\
& \multicolumn{5}{c}{$1-42$ days of age } \\
Weight gain, g & 2558 & 2558 & 2505 & 2621 & 4.22 \\
Feed intake, g & 4250 & 4083 & 4063 & 4123 & 2.81 \\
Feed:gain ratio & 1.80 & 1.74 & 1.76 & 1.70 & 4.88 \\
Livability rate, \% & 97.46 & 97.77 & 97.14 & 97.17 & 2.35 \\
\hline
\end{tabular}

$\mathrm{CV}=$ Coefficient of variation.

Cuadro 7. Rendimiento de vísceras comestibles, en canal y cortes de pollos de engorda con dietas con diversos niveles de polen de abeja (BP)

Table 7. Yields for carcass, cuts and edible viscera of broiler chicks fed diets containing levels of bee pollen (BP)

\begin{tabular}{lrrrrr}
\hline & \multicolumn{4}{c}{ BP level (\%) } & \\
\cline { 2 - 4 } & \multicolumn{1}{c}{0.0} & \multicolumn{1}{c}{0.5} & 1.0 & 1.5 & CV (\%) \\
\hline Carcass & 803.9 & 824.9 & 867.1 & 849.4 & 5.33 \\
Breast & 275.3 & 278.4 & 311.0 & 294.6 & 4.42 \\
Thigh and drumstick & 222.5 & 236.4 & 227.9 & 242.7 & 5.72 \\
Liver & 21.8 & 22.1 & 21.4 & 20.2 & 4.04 \\
Heart & 6.3 & 6.3 & 5.7 & 5.8 & 3.68 \\
Gizzard & 16.2 & 18.4 & 12.4 & 18.1 & 3.27 \\
Pancreas $^{1}$ & 1.8 & 1.6 & 2.1 & 2.2 & 3.09 \\
\hline
\end{tabular}

$\mathrm{CV}=$ Coefficient of variation.

1 Quadratic effect $\left(\hat{Y}=0.18-0.020 x+0.034 x^{2} ; R^{2}=0.42\right)$. 
productivo de las aves en el periodo de 1 a 42 días de edad (Cuadro 6) y los rendimientos de la canal, cortes de primera y las vísceras comestibles (Cuadro 7). Hubo efecto cuadrático en el tamaño del páncreas $(P<0.03)$ en que se obtuvieron los valores más altos con la inclusión de $1.5 \%$ de PA.

El PA tuvo un efecto de segundo grado $(P<0.014)$ en la cantidad de amoniaco volatilizado de la cama de pollo de 42 días de crianza, pero los otros parámetros no fueron afectados $(P>0.05)$ a los 21 y 42 días (Cuadro 8).

\section{DISCUSIÓN}

Los valores más altos de DAMS, DAEE, retención de $\mathrm{Ca}, \mathrm{EMA}$ y EMAn se obtuvieron con la inclusión de $1.5 \%$ con aumento del $4.09 \%$ en DAMS, $2.31 \%$ en CDEE y $12.06 \%$ en la retención de $\mathrm{Ca} ; 9.41 \%$ en el valor EMA y $9.63 \%$ en el valor EMAn en comparación con el tratamiento sin PA, lo que indica su efecto beneficioso, al ser rica en varios nutrientes tales
AMEn were obtained with the inclusion of $1.5 \%$. Increase of $4.09 \%$ in ADDM, $2.31 \%$ in CDEE and $12.06 \%$ in Ca retention, $9.41 \%$ in the AME value and $9.63 \%$ in the AMEn value compared with the treatment without BP. So BP has a beneficial effect because is rich in several nutrients such as amino acids, fats, vitamin C, oligoelements, enzymes and flavonoids, that are antioxidant factors also important for cell and intestinal mucosa differentiation(4), responsible for nutrient absorption.

Furthermore, the presence of digestive enzymes in $\mathrm{BP}(2)$ and increase in the number of lactobacilli that acidify the intestine and increase mineral absorption and amylase, protease and phytate secretion(12) collaborated to improve nutrient use by the birds.

At $21 \mathrm{~d}$ of age, the highest values for duodenum villus height and perimeter were obtained with the inclusion of $1.05 \%$, in both parameters. In the jejunum, at $21 \mathrm{~d}$ of rearing, the greatest values of villus height, perimeter and width and surface absorption were obtained by including

Cuadro 8. Calidad de la cama de pollos de engorda alimentados con dietas con diversos niveles de polen de abeja (BP)

Table 8. Litter quality of broiler chick fed diets containing levels of bee pollen (BP)

\begin{tabular}{|c|c|c|c|c|c|}
\hline & \multicolumn{4}{|c|}{ BP level (\%) } & \multirow[b]{2}{*}{ CV (\%) } \\
\hline & 0.0 & 0.5 & 1.0 & 1.5 & \\
\hline \multicolumn{6}{|c|}{$21 \mathrm{~d}$ of age } \\
\hline $\mathrm{pH}$ & 6.29 & 6.19 & 5.93 & 6.03 & 6.31 \\
\hline Volatilized ammonia, ppm & 12.62 & 14.21 & 10.15 & 16.70 & 10.55 \\
\hline Dry matter, \% & 95.28 & 95.69 & 94.44 & 93.93 & 1.47 \\
\hline Total nitrogen, \% & 2.28 & 2.22 & 2.03 & 2.36 & 5.32 \\
\hline Total phosphorus, \% & 0.78 & 0.75 & 0.73 & 0.74 & 5.91 \\
\hline \multicolumn{6}{|c|}{$42 \mathrm{~d}$ of age } \\
\hline $\mathrm{pH}$ & 9.07 & 8.82 & 8.97 & 9.04 & 2.44 \\
\hline Volatilized ammonia, ppm 1 & 240.79 & 114.26 & 175.77 & 118.33 & 6.73 \\
\hline Dry matter, \% & 70.79 & 60.88 & 68.26 & 65.08 & 7.38 \\
\hline Total nitrogen, \% & 2.64 & 2.77 & 2.51 & 2.64 & 4.75 \\
\hline Total phosphorus, \% & 1.45 & 1.50 & 1.56 & 1.52 & 5.49 \\
\hline
\end{tabular}


como aminoácidos, grasas, vitamina C, oligoelementos, enzimas y flavonoides, que son factores antioxidantes también importantes para la célula y diferenciación intestinal de la mucosa(4), responsable de la absorción de nutrientes.

Además, la presencia de enzimas digestivas en el PA(2) y el aumento en el número de lactobacilos que acidifican el intestino y aumentan la absorción de minerales y amilasa, proteasa y la secreción de fitato(12), colaboraron para mejorar el uso de nutrientes por las aves.

A los 21 días de edad, los valores más grandes de altura de las vellosidades del duodeno y el perímetro se obtuvieron con la inclusión de $1.05 \%$, en ambos parámetros. En el yeyuno, a los 21 días de cría, los mayores valores de la altura de las vellosidades, el perímetro y la absorción de la anchura y la superficie se obtuvieron mediante la inclusión de 1.06, 1.15, 1.50 y $1.50 \%$ PA, respectivamente. En el íleon, a los 42 días de cría, los valores más altos de altura de las vellosidades y el perímetro se obtuvieron con la inclusión de 0.90 y 0.86 $\%$ PA en las dietas de aves.

El polen de abejas contiene aminoácidos, vitamina $C$ y oligoelementos que son nutricionalmente beneficiosos para el desarrollo de las células del tejido, la proliferación celular epitelial y la diferenciación y el crecimiento de microbiota intestinal. Esto resulta en un aumento de la superficie intestinal para la absorción de nutrientes $(4,7)$. El PA presentó un efecto trófico sobre el duodeno, el yeyuno y el íleon en niveles que van desde 0.86 hasta $1.50 \%$. La altura de las vellosidades y el perímetro disminuyeron en el yeyuno a los 21 días y en el íleon a los 42 días con la inclusión de $1.5 \%$, y como el intestino es el sitio principal para la digestión y absorción de nutrientes, pueden haber sido un reflejo de la mayor digestibilidad de los nutrientes con el $1.5 \%$ de PA, lo que reduciría la necesidad de tejido de la mucosa(13).

Wang et a/(6) estudiaron la inclusión de 0 y $1.5 \%$ de PA en dietas de pollo de engorda
$1.06,1.15,1.50$ and $1.50 \% \mathrm{BP}$, respectively. In the ileum, at $42 \mathrm{~d}$ of rearing, the highest villus height and perimeter values were obtained with the inclusion of 0.90 and $0.86 \% \mathrm{BP}$ in the bird diets.

BP contains amino acids, vitamin $\mathrm{C}$ and oligoelements that are nutritionally beneficial for the development of tissue cells, epithelial cell proliferation and differentiation and intestinal microbiota growth. This results in an increase in the intestine surface for nutrient absorbance $(4,7)$. The BP presented a trophic effect on the duodenum, jejunum and ileum at levels ranging from 0.86 to $1.50 \%$. The villus height and perimeter decreased in the jejunum at $21 \mathrm{~d}$ and in the ileum at $42 \mathrm{~d}$ with the inclusion of $1.50 \%$. As the intestine is the main site for nutrient digestion and absorption, it may have been a reflection of the greater nutrient digestibility with $1.50 \%$ BP included that would reduce the need for tissue in the mucosa(13).

Wang et a/(6) studied the inclusion of 0 and $1.5 \%$ BP in broiler chick diets and reported that the villi in the duodenum of birds that ingested BP were longer up to $21 \mathrm{~d}$ and up to $14 \mathrm{~d}$ in the jejunum and ileum. The crypts were deeper in the birds that received BP up to $14 \mathrm{~d}$ in all the intestinal segments, due to the trophic action of BP that induced cell proliferation and differentiation in the intestinal mucosa, because height is directly proportional to the number of epithelial cells.

The lack of effect on the productive performance could be due to the fact that corn and soybean meal are ingredients with high nutritive value and therefore the reduction in the levels of crude protein and metabolizable energy may not have been sufficient to allow the BP to have a greater effect. Furthermore, the good housing conditions of the birds may have collaborated to lack of challenge and consequently difference in the performance results.

Different results were reported by Wang et al(6) who studied the effect of including $1.5 \%$ BP in 
e informaron que las vellosidades en el duodeno fueron más grandes a los 21 días y mayores a los 14 días en el yeyuno e íleon. Las criptas fueron más profundas en las aves que recibieron PA hasta 14 días en todos los segmentos del intestino, debido a la acción trófica del polen, que induce la proliferación y la diferenciación celular en la mucosa intestinal, ya que la altura es directamente proporcional al número de células epiteliales.

La falta de efecto en el comportamiento productivo podría ser debido al hecho de que el maíz y la harina de soya son ingredientes con alto valor nutritivo, y por lo tanto la reducción en los niveles de proteína cruda y energía metabolizable puede no haber sido suficiente para permitir que el PA tuviera un mayor efecto. Por otra parte, las buenas condiciones de alojamiento de las aves pueden haber colaborado a la falta de desafío y, por consiguiente diferenciar los resultados de rendimiento.

Diferentes resultados fueron reportados(6), con inclusiones de $1.5 \%$ de la PA en la dieta de pollos de engorda, con pesos semanales hasta los 42 días de la crianza, mencionando que el peso corporal de estas aves fue mayor cada semana que en las aves del tratamiento control, y este hecho se atribuyó al efecto trófico del polen en la mucosa intestinal.

Los rendimientos en canal, cortes primarios y vísceras comestibles no se vieron afectados, pero el páncreas aumentó con la inclusión de $1.5 \%$ de PA. El aumento en el tamaño del páncreas puede haber contribuido a una mayor digestibilidad de proteína cruda y mayor energía metabolizable, debido a la secreción de sus enzimas digestivas.

Song et al(7) mencionaron que el hígado y el páncreas fueron mayores en pollos de engorda que recibieron PA hasta la cuarta y quinta semana de edad, respectivamente, un efecto no observado en el presente experimento. El hígado es la glándula relacionada con el the diet of broiler chicks weighed weekly up to $42 \mathrm{~d}$ of rearing. They observed that the body weight of these birds was greater every week than the birds of the control treatment, attributed to the trophic effect of BP on the intestinal mucosa of these birds.

The yields for carcass, noble cuts and edible viscera were not affected but the pancreas was increased with including $1.5 \%$ BP. The increase in the pancreas may have contributed to greater crude protein digestibility and greater metabolizable energy, due to the secretion of its digestive enzymes.

Song et al(7) also assessed the effects of PA on broiler chick liver and pancreas weight and observed that the liver and pancreas were greater in broiler chicks that received BP up to the fourth and fifth weeks of age, respectively, an effect not observed in the present experiment. The liver is the gland related to nutrient metabolim and the pancreas secretes various digestive enzymes. However, other authors(14) used $0.2 \%$ bee pollen polysaccharides in chicken diet and observed that there was no influence on the carcass, breast and thigh yields in the sixth or eighth week of age.

With regard to the volatilized ammonia, BP presented a low $\mathrm{pH}$, around 4.0 and 5.0 (3) and the ammonia-producing bacteria may have been inhibited and acidophyle bacteria proliferation stimulated that are beneficial to organisms. Some research(15), indicate that including BP in animal diet helped to inhibit pathogenic bacteria in the intestines and accelerated proliferation of the normal microbiota. In studies with rats submitted to chronic stress(16) observed reduction in the bifidobacteria and increase in the enterobacteriaceae and enterococcus, indicating a situation of dysbiosis in the intestinal macrobiota. However, in rats that received feed supplemented with $0.50 \%$ BP there was no reduction in the bifidobacteria even in stress state, showing a protective effect of BP against dysbiosis. 
metabolismo de nutrientes y el páncreas secreta diversas enzimas digestivas. Sin embargo, otros autores(14) utilizaron $0.2 \%$ de polisacáridos de polen de abeja en la dieta de pollo y observaron que no hubo influencia sobre el rendimiento de la canal, la pechuga y de los muslos en la sexta u octava semana de edad.

Con respecto al amoniaco volatilizado, el polen presentó un pH bajo, alrededor de 4.0 y 5.0(3); las bacterias productoras de amoníaco pueden haber sido inhibidas y la proliferación de bacterias acidofílicas estimuladas (que son beneficiosas para los organismos). Algunos estudios indican que incluyendo PA en la dieta de los animales ayudó a inhibir bacterias patógenas en los intestinos y la proliferación acelerada de la microbiota normal(15). En estudios con ratas sometidas a estrés crónico(16) se observó una reducción en las bifidobacterias y aumento de enterobacterias y enterococos, lo que indica una situación de disbiosis en la macrobiota intestinal. Sin embargo, en las ratas que recibieron alimento suplementado con $0.5 \%$ de PA no hubo reducción en las bifidobacterias incluso en estado de estrés, que muestra un efecto protector del polen contra disbiosis.

\section{CONCLUSIONES E IMPLICACIONES}

El polen de abeja, cuando se incluye en $1.5 \%$ de la dieta para pollos de engorda, mejora la digestibilidad de los nutrientes y aumenta la altura y el perímetro de las vellosidades intestinales, pero no mejoró el rendimiento productivo.

\section{AGRADECIMIENTOS}

Al Conselho Nacional de Desenvolvimiento Científico e Tecnológico - CNPq por el financiamiento de la investigación científica y a BRFoods S.A. por la donación de las aves para el experimento de digestibilidad.

\section{CONCLUSIONS AND IMPLICATIONS}

Bee pollen, when included at $1.5 \%$ diet for broiler chicks, improves nutrient digestibility and increases intestinal villus height and perimeter but did not improve productive performance.

\section{ACKNOWLEDGEMENTS}

To Conselho Nacional de Desenvolvimento Científico e Tecnológico - CNPq by the scientific research funding and BRFoods S.A. for donating the birds for the digestibility experiment.

\section{End of english version}

\section{LITERATURA CITADA}

1. Carpes ST, Prado A, Moreno IAM, Mourão GB, Alencar SM, Masson ML. Avaliação do potencial antioxidante do pólen apícola produzido na região Sul do Brasil. Quim Nova 2008; (31):1660-1664.

2. Wang J, Gu Y, Li S, Fang ZS, Feng BM. Effect of bee pollen on histological structure of digestive organs of layer. J Anhui Univ Sci Tech 2006;(20):1-6.

3. Marchini LC, Reis VDA, Moreti ACCC. Composição físicoquímica de amostras de pólen coletado por abelhas africanizadas. Cienc Rural 2006;(36):949-953.

4. Wang J, Song Y, Li S, Wang Q. Effect of bee pollen on development of small intestine in broilers. Chin J Vet Sci Tech 2005; (35):484-488.

5. Neves LC, Alencar SM, Carpes ST. Determinação da atividade antioxidante e do teor de compostos fenólicos e flavonóides totais em amostras de pólen apícola de Apis mellifera. Braz J Food Technol 2009;(12):107-110.

6. Wang J, Li S, Wang Q, Xin B, Wang H. Trophic effect of bee pollen on small intestine in broilers chickens. J Med Food 2007; (10):276-280.

7. Song Y, Wang J, LI S, Shang C. Effect of bee pollen on the development of digestive gland of broilers. China Anim Husband Vet Med 2005;(37):14-17.

8. Pan K, Sun H, Gao Z. Effect of bee pollen polysaccharide on growth performance of broilers and chemical compositions of muscle. Feed Ind 2006;(27):39-41.

9. Rostagno HS, Albino LFT, Donzele JL, Gomes PC, Oliveira RF, Lopes DC, et al. Tabelas brasileiras para aves e suínos: composição de alimentos e exigências nutricionais. $2^{a}$ ed. Viçosa, Brasil: Editora Universitária; 2011. 
María Cristina de Oliveira, et al. / Rev Mex Cienc Pecu 2015;6(3):263-276

10. Silva DJ, Queiroz AC. Análise de alimentos: métodos químicos e biológicos. 3a ed. Viçosa, Brasil: Editora Universitária; 2002.

11. Oliveira MC, Ferreira HA, Cancherini LC. Efeito de condicionadores químicos sobre a qualidade da cama de frango. Arq Bras Med Vet Zoot 2004;(56):536-541.

12. Taheri H, Tabandeh F, Moravej H, Zaghari M, Shivazad M, Shariati P. Potential probiotic of Lactobaillus johnsonii LT171 for chicken nutrition. Afr J Biotechnol 2009;(8):5833-5837.

13. Gilbert C, Acamovic T, Bedford MR. The effects of lupin inclusion with or without enzyme supplementation on the morphology of the posterior tract gastrointestinal. Brit Poult Sci 2001:(42):S90.

14. Ke P, Han S, Zhihui G. Effect of bee pollen polysaccharide on growth performance of broilers and chemical composition of muscle. Feed Ind 2006;(27):39-41.

15. Sun Y, Wang MJ, Bu SJ. The pharmacological health effect and the veterinary clinical application of bee pollen. Vet Pharm Feed Addit 2009;(14):27-28.

16. Bai SM, Liu Y, Gao AX. Effects of pine pollen on the gut microbiology of rats under chronic stress. Asia Pac J Clin Nutr 2006; (15):S142. 